\title{
Understanding Nanotechnology and Nanoscience for National Development
}

\author{
Usman Maijama'a Kallamu \\ Department of Mechanical Engineering, The Federal Polytechnic \\ Damaturu, Yobe State, Nigeria
}

\section{Doi:10.5901/ajis.2013.v2n13p103}

\begin{abstract}
The emergence of nanoscience and nanotechnology has opened a myriad possibilities to revolutionize a wide range of fields ranging from cosmetics to space technologies; there are also important contributionsto diagnostics and therapeutic practices, especially for treating cancer. Nanoscience is also making a difference in many areas of engineering and technology, such as energy environmental pollution control, textile, automobile and electronics. Nanoscience and nanotechnology combine the essence of knowledge in many fields of science and are truly interdisciplinary. There is little doubt that the next generation of citizens will be the beneficiaries of the applications of nanoscience and nanotechnology in their daily life. It is, therefore, vital to have adequate awareness of this important field, irrespective of the specialization or profession that one may choose.
\end{abstract}

\section{Introduction}

One of the scientific research areas in recent years, with astonishing developments, has been nano-mechanics, as an important part of nano-technology. This relatively new field of mechanical sciences is becoming more and more responsive to the practical needs of nano-technology. Fluids with nanoparticles suspended in them are called nanofluids. This term was coined by Choi in 1995 at Argonne National laboratory of USA [1]. Nanofluids are tough to be the next-generation heat transfer fluids, and they offer exciting possibilities due to their enhanced heat transfer performance compared to ordinary fluids. The advantages of these nanofluids are (1) better stability compared to those fluids containing micro-or milli-sized particles and (2) higher thermal conductive capability than the base fluids themselves. Nanofluids proposed for various uses in important fields such as electronics, transportation, medical, and HVAC [2]. Hence, there is a need for fundamental understanding of the heat transfer behavior of nanofluids in order to exploit their potential benefits and applications.

\subsection{History and Scope}

The word 'nano' is to a Greek prefix meaning dwarf or something very small and depicts one billionth (10-9) of a unit (refer Table 1.1). Nanomaterials, therefore, refer to the class of materials with at least one of the dimensions in the nanometric range. How small is a nanocrystal? For an immediate comparison, a nanometre represents a dimension about a few tens of thousand times thinner than human hair, In the case of polycrystalline materials, the grain size is typically of the order of $1-100$ microns ( 1 micron $=10-6 \mathrm{~m}$ ). Nanocrystalline materials have a grain size of the order of 1-100 nm, and are therefore 100-1000 times smaller than conventional grain dimensions. However,compared to the size of an atom (0.2-0.4 nm in diameter), nanocrystalline grains are still significantly big.For example, a nanocrystal of size $10 \mathrm{~nm}$ contains over a hundred thousand atoms 
(assuming a spherical nanograin of $10 \mathrm{~nm}$ and atomic diameter of $0.2 \mathrm{~nm}$ ), large enough to exhibit bulk properties, i.e., properties different from those of single atoms or clusters. As the dimensions reduce to below 50-100 nm, they can no longer be treated as infinite systems and the resultant boundary effects lead to fascinating and useful properties, which can be explored and tailored for a variety of structural and functional applications. In principle, one cannot define an exact dimension for the grain size below which materials can be classified as 'nano'. This is because it is subjective and depends on the application or end property of interest. Most electronic and optical properties vary when the grain size is reduced typically below $10 \mathrm{~nm}$. However, their mechanical, chemical and many physical properties begin to vary significantly from bulk below 50-100 nm. Hence, nanomaterials may be classified as those materials which have at least one of their dimensions in the nanometric range, below which there is significant variation in the property of interest compared to microcrystalline materials. Nanomaterials can be metals, ceramics, polymers or composites. Nanotechnology is an umbrella term for many areas of research dealing with objects that have one of their dimensions

Table 1.1 The world of small dimensions

Number
0.1
0.01
0.001
0.000001
0.000000001
0.000000000001
0.000000000000001
0.000000000000000001
0.000000000000000000001
0.000000000000000000000001
4 Nanoscience and Nanotechnology.

\section{Applications of Nanofluids}

\subsection{Heat transfer Intensification}

Since the origination of the nano fluid concept about a decade ago, the potentials of nanofluids in heat transfer application have attracted more and more attention. Up to now, there are some review papers, which present over views of various aspects of nanofluids [1, 3-6, ], including preparation and characterization, techniques for the measurements of thermal conductivity, theory and model, thermo physical properties, convective heat transfer.

\subsubsection{Electronic applications}

Due to higher density of chips, design of electronic components with more compact makes heat dissipation more difficult. Advanced electronic devices face thermal management challenges from the high level of heat generation and the reduction of available surface area for heat removal so, the reliable thermal management system is vital for the smooth operation of the advanced electronic devices. In general, there are two approaches to improve the heat removal for electronic equipment. One is to find an optimum geometry of cooling devices; another is to increase the heat transfer capacity. Nanofluids with higher thermal conductivities are predicated convective heat transfer coefficients compared to those of base fluids. 


\subsubsection{Transportation}

Nanofluids have great potentials to improve automotive and heavy-duty engine cooling rates by increasing the efficiency, lowering the weight and reducing the complexity of thermal management systems. The improved cooling rates for automotive and truck engines can be used to remove more heat from higher horsepower engines with the same size of cooling system. Alternatively, it is beneficial to design more compact cooling system with smaller and lighter radiators.

\subsubsection{Industrial cooling applications}

The application of nanofluids in industrial cooling will result in great energy savings and emissions reductions. For US industry, the replacement of cooling and heating water with nanofluids has the potential to conserve 1 trillion Btu of energy [4, 6]. For the US electric power industry, using nanofluids in closed loop cooling cycles could save about 10-30 trillion Btu per year (equivalent to the annual energy consumption of about 50,000-150,000 households). The associated emissions reductions would approximately 5.6 million metric tons of carbon dioxide, 8,600 metric tons of nitrogen oxides, and 21,000 metric tons of sulfur dioxide [5].

\subsubsection{Heating building and reducing pollution}

Nanofluids can be applied in the building heating systems. Kulkarni et al. evaluated how they perform heating buildings in cold regions [8]. In cold regions, it is a common practice to use ethylene or propylene glycol mixed with water in different proportions as a heat transfer fluids.

\subsubsection{Nuclear systems cooling}

The Massachusetts institute of Technology has established an interdisciplinary center for nanofluid technology for the nuclear energy industry. The researchers are exploring the nuclear applications of nanofluids, a specifically the following three [9, main reactor coolant for pressurized water reactors (PWRs). It could enable significant power upgrates in current and future PWRs, thus enhancing their economic performance. Specifically, the use of nanofluids with at least $32 \%$ higher critical heat flux (CHF) could enable a $20 \%$ power density uprate in current plants without changing the fuel assembly design and without reducing the margin to CHF; 2) coolant for the emergency core cooling systems (ECCSs) of both PWRs and boiling water reactors. The use of a nanofluid in the ECCS accumulators and safety injection can increase the peak-cladding-temperature margins (in the nominal-power core) or maintain them in uprated cores if the nanofluid has a higher postCHF heat transfer rate; 3) coolant for in-vessel retention of the molten core during severe accidents in high-power-density light water reators. It can increase the margin to vessel breach by $40 \%$ during severe accidents in high-power density systems such as Westinghouse APR1000 and the Korean APR1400.

\subsubsection{Space and defense}

Due to the restriction of space, energy and weight in space station and aircraft, there is a strong demand for high efficient cooling system with smaller size. You et al. [7] and Vassalo et al. [1] have reported order of magnitude increases in the critical heat flux in pool boiling with nanofluids compared to the base fluids alone.

\subsection{Mass transfer enhancement}

Several researches have studied the mass transfer enhancement of nanofluids. Kim et al. initially 
examined the effect of nanoparticles on the bubble type absorption for $\mathrm{NH}_{3} / \mathrm{H}_{2} \mathrm{O}$ absorption system [2]. The addition of nanoparticles enhances the absorption performance up to 3.21 times. Then they visualized the bubble behavior during the $\mathrm{NH}_{3} / \mathrm{H}_{2} \mathrm{O}$ absorption process and studied the effect of nanoparticles and surfactants and nanoparticles on the absorption characteristics [3]. The results show that the addition of surfactants and nanoparticles improved the absorption performance up to 5.32 times. The addition of both surfactants and nanoparticles improved the absorption performance up to 5.32 times. The addition of both surfactants and nanoparticles enhanced significantly the absorption performance during the ammonia bubble absorption process.

\subsection{Energy applications}

For energy application of nanofluids, two remarkable properties of nanofluids are utilized, one is the higher thermal conductivities of nanofluids enhancing the heat transfer, another is the absorption properties of nanofluids.

\subsubsection{Energy storage}

The temporal difference of energy source and energy needs made necessary the development of storage system. The storage of thermal energy in the form of sensible and latent heat has become an important aspect of energy management with the emphasis on efficient use and conservation of the waste heat and solar energy in industry and buildings [7]. Latent heat storage is one of the most efficient ways of storing thermal energy.

\subsubsection{Solar absorption}

Solar energy is one of the best sources of renewable energy with minimal environmental impact. The conventional direct absorption solar collector is a well established technology, and it has been proposed for a variety of applications such as water heating; however the efficiency of these collectors is limited by the absorption properties of the working fluid, which is very poor for typical fluids used in solar collectors.

\subsection{Mechanical applications}

Why nanofluids have great friction reduction properties? Nanoparticles in nanofluids form a protective film with low hardness and elastic modules on the worn surface can be considered as the main reason that some nanofluids exhibit excellent lubricating properties.

Magnetic fluids are kinds of special nanofluids. Magnetic liquid rotary seals operate with no maintenance and extremely low leakage in a very wide range of applications, and it utilizing the proprety magnetic properties of the magnetic nanoparticles in liquid.

\subsubsection{Friction reduction}

Advanced lubricants can improve productivity through energy saving and reliability of engineered systems. Tribological research heavily emphasizes reducing friction and wear. Nanoparticles have attracted much interest in recent years due to their excellent load-carrying capacity, good extreme pressure and friction reducing properties.

\subsubsection{Magnetic sealing}

Magnetic fluids (Ferromagnetic fluids) are kinds of special nanofluids. They are stable colloidal suspensions of small magnetic particles such as magnetite $\left(\mathrm{Fe}_{3} \mathrm{O}_{4}\right)$. The properties of the magnetic 
nanoparticles, the magnetic component of magnetic nanofluids, may be tailored by varying their size and adapting their surface coating in order to meet the requirements of colloidal stability of magnetic nanofluids with non-polar and polar carrier liquids [4].

\subsection{Biomedical application}

\subsubsection{Antibacterial activity}

Organic antibacterial materials are often less stable particularly at high temperatures or pressures. As a consequence, inorganic materials such as metal and metal oxides have attracted lots of attention over the past decade due to their ablilty to withstand harsh process conditions. The antibacterial behavior of $\mathrm{ZnO}$ nanofluids shows that the $\mathrm{ZnO}$ nanofluids have bacteriostatic activity against [8].

\subsubsection{Nanodrug delivery}

Over the last few decades, colloidal drug delivery systems have been developed in order to improve the efficiency and the specificity of drug action [1]. The small size, customized surface, improved solubility, and multi-functionality of nanoparticles open many doors and create new biomedical applications. The novel properties of nanoparticles offer the ability to interact with complex cellular functions in new ways [1].

\subsection{Other applications}

\subsection{Intensify micro reactors}

The discovery of high enhancement of heat transfer in nanofluids can be applicable to the areas of process intensification of chemical reactors through integration of the functionalities of reaction and heat transfer in compact multifunctional reactors. Fan et al. studied reactor-heat exchanger [2]. The overall heat transfer coefficient increase was up to $35 \%$ in the steady state continuous experiments. This resulted in a closer temperature control in the reaction of selective reduction of an aromatic aldehyde by molecular hydrogen and very rapid change in the temperature of reaction under dynamic reaction control.

\subsubsection{Nanofluids as vehicular brake fluids}

A vehicle's kinetic energy is dispersed through the heat produced during the process of braking and this is transmitted throughout the brake fluid in the hydraulic brake], and now there is a higher demand for the properties of brake oils. Copper-oxide and aluminum oxide based brake nanofluids were manufactured using the arc-submerged nanoparticle synthesis system and plasma charging arc system, respectively [1].

\subsubsection{Nanofluids based microbial fuel cell}

Microbial fuel cells (MFC) that utilized the energy found in carbohydrates, proteins and other energy rich natural products to generate electrical power have a promising future. The excellent performance of MFC depends on electrodes and electron mediator. Sharma et al. constructed a novel microbial fuel cell (MFC) using novel electron mediators and CNT based electrodes [4]. The novel mediators are nanofluids which were prepared by dispersing nanocrystalline platinum anchored CRTs in water. 


\subsubsection{Nanofluids with unique optical properties.}

Optical filters are used to select different wavelengths of light. The ferro fluid based optical filter has tunable properties. The desired central wavelength region can be tuned by an external magnetic field, Philip et al. developed a ferrofluid based emulsion for selecting different bands of wavelengths in the UV, visible and IR regions [1).

\section{Preparation of Nanofluids}

Preparation of nanofluids is the first key step in experimental studies with nanofluids. Nanofluids are not simply liquid-solid mixtures. Some special requirements are essential e.g. even and stable suspension, durable suspension, negligible agglomeration of particles, no chemical change of the fluids, e.t.c Nanofluids are produced by dispersing nanometer-scale solid particles into base liquids such as water, ethylene glycol (EG), oils, e.t.c. In the synthesis of nanofluids, agglomeration is a major problem. There are mainly two techniques used to produce nanofluids: the single-step and the two-step method. The single-step direct evaporation approach was developed by Akoh et al. [4] and is called the VEROS (vacuum Evaporation onto a Running Oil Substrate) technique. The original idea of this method was to produce nanoparticles, but it is difficult to subsequently separate the particles from the fluids to produce dry nanoparticles. A modified VEROS process was proposed by Wagener et al. [5]. They employed high pressure magnetron sputtering for the preparation of suspension with metal nanoparticles such as Ag and Fe. Eastman et I. [6] developed a modified VEROS technique, in which $\mathrm{Cu}$ vapor is directly condensed into nanoparticles by contact with a flowing low-vapor-pressure liquid (EG).

Zhu et al. [7] presented a novel one-step chemical method for preparing copper nanofluids by reducing $\mathrm{CuSO}_{4} \cdot 5 \mathrm{H}_{2} \mathrm{O}$ with $\mathrm{NaH}_{2} \mathrm{PO}_{2} \cdot \mathrm{H}_{2} \mathrm{O}$ in ethylene glycol under microwave irradiation. Results showed that the addition of $\mathrm{NaH}_{2} \mathrm{PO}_{2} \cdot \mathrm{H}_{2} \mathrm{O}$ and the adoption of microwave irradiation are two significant factors which affect the reaction rate and the properties of $\mathrm{Cu}$ nanofluids.

A vacuum-SANSS (submerged arc nanoparticles synthesis system) method has been employed by Lo et al. [8] to prepare Cu-based nanofluids with different dielectric liquids such as de-ionized water, with 30\%, 50\%, $70 \%$ volume solutions of ethylene glycol and pure ethylene glycol. They found that the different morphologies, which are obtained, are mainly influenced and determined by the thermal conductivity of the dielectric liquids. $\mathrm{CuO}, \mathrm{Cu}_{2} \mathrm{O}$, and $\mathrm{Cu}$ based nanofluids also can be prepared by this technique efficiently. An advantage of the one-step technique is that nanoparticle agglomeration is minimized, while the disadvantage is that only low vapor pressure fluids are compatible with such a process. Recently, a Ni nanomagnetic fluid was also produced by Lo et al. [7] using the SANSS method.

The two-step method is extensively used in the synthesis of nanofluids considering the available commercial nanopowders supplied by several companies. In this method, nanoparticles was first produced and then dispersed the base fluids. Generally, ultrasonic equipment is used to intensively disperse the particles and reduce the agglomeration of particles. For example, Eastman et al. [6], Lee et al. [10], and Wang et al. [1] used this method to produce Al2O3 nanofluids. Also, Murshed et al. [2] prepared $\mathrm{TiO}_{2}$ suspension in water using the two-step method. Other nanoparticles reported in the literature are gold $(\mathrm{Au})$, silver $(\mathrm{Ag})$, silica and carbon nanotubes: $A s$ compared to the single-step method, the two-step technique works well for oxide nanoparticles, while it is less successful with metallic particles.

Except for the use of ultrasonic equipment, some other techniques such as control of $\mathrm{pH}$ or addition of surface active agents, are also used to attain stability of the suspension of the nanofluids against sedimentation. These methods change the surface properties of the suspended particles and thus suppress the tendency to form particle clusters. It should be noted that the selection of surfactants should depend mainly on the properties of the solutions and particles. Xuan and $\mathrm{Li}$ [3] chose salt and oleic acid as the dispersant to enhance the stability of transformer oil-Cu 
and water-Cu nanofluids, respectively. Oleic acid and cetyltrimethyle ammonium bromide (CTAB) surfactants were used by Murshed et al. [2] to ensure better stability and proper dispersion of $\mathrm{TiO}_{2}$-water nanofluids. Sodium dodecyl sulfate (SDS) was used by Hwang et al. [4] during the preparation of water-based MWCNT nanifluids since the fibers are entangled in the aqueous suspension.

In general, methods such as change of $\mathrm{pH}$ value, addition of dispersant, and ultrasonic vibration aim at changing the surface properties of suspended particles and suppressing formation of particles cluster to obtain stable suspensions. However, the addition of dispersants can affect the heat transfer performance of the nanofluids, especially at high temperature.

\subsection{Nanoparticles}

In nanotechnology, a particle is defined as a small object that behaves as a whole unit with respect to its transport and properties. Particles are further classified according to diameter. Coarse particles cover a range between 10,000 and 2,500 nanometers. Fine particles are sized between 2,500 and 100 nanometers. Ultrafine particles or nanoparticles are sized between 1 and 100 nanometers. The reason for this double name of the same object is that, during the 1970-80's, when the first through fundamental studies with "nanoparticles" were underway in the USA (by Granqvist and Buhram) and Japan, (within an ERATO Project they were called "ultrafine particles" (UFP). However, during the 1990s before the National Nanotechnology I nitiative was launched in the USA, the new name, "nanoparticle" had become fashionable (see, for example the same senior author's paper 20 years later addressing the same issue, lognormal distribution of sizes). Nanoparticles may or may not exhibit size-related properties that differ significantly from those observed in fine particles or bulk materials.(5) Although the size of most molecules would fit into the above outline, individual modules are usually not referred to as nanoparticles.

\section{Clusters and Magic Numbers}

When atoms come together, they initially form two-dimensional clusters. When more atoms join the cluster, they become three-dimensional with topographically close-packed arrangements, before taking up a crystal structure. It has been found that clusters of certain critical sizes, i.e., clusters with a certain number of atoms in the group, are more stable than others. Such clusters are stabilized either by geometric or electronic considerations. Magic number is defined as the number of atoms in the clusters of critical sizes with higher stability. This effect was initially observed in gaseous metal atoms, in the early 1980s. Subsequently, a similar effect was also noticed during the condensation of atoms from a vapour phase on a substrate surface, for example, during thin film deposition. Magic numbers based on electronic shells were first observed in mass spectra of alkali' metal clusters. The stability of such clusters continuous function. It was seen that for some specific number of

\subsection{Challenges and Future Prospects}

With so many achievements already realised, it is perhaps pertinent to ask "Where is nanotechnology heading"? It is envisaged that nanotechnology will lead to tiny robotic devices, utilizing nanoelectronics, sensors and MEMS/NEMS for in-vivo monitoring and diagnosis of electrooptic deficiencies and malfunctions of human systems. Yet, the current applications of nanotechnology are much more mundane: stain-resistant trousers, better sun creams, tennis rackets reinforced with carbon nanotubes! There is a huge gap between what nanotechnology is believed to have promised and what it has actually delivered so far, In his book, Engines of Creation: The Coming Era of Nanotechnology, published in 1986, Drexler imagined sophisticated nanoscale machines that could operate with atomic precision. He envisaged a particular way of 
achieving nanotechnology, which involved using hard materials like diamond to fabricate complex nanoscale structures by moving reactive molecular fragments into position. His approach was essentially mechanical, whereby tiny gears and bearings are integrated to make tiny robot factories, probes and vehicles. Drexler postulated that since nanoscale machines are expected to be extensively employed in biological systems and would be synthesized in significantly large quantities under ambient conditions, it should be possible to discover the growth conditions to synthesize them for a variety of other applications as well. The beauty of nanotechnology is that it is truly multidisciplinary, re-unifying the common threads between science, engineering and technology.It is so vivid, with possibilities left only to the constraint of perhaps human imagination. With a little exaggeration, it seems possible that materials with any desired physical, chemical or electronic properties can be tailor-made by playing with the nano-dimensions. The next generation is going to be directly or indirectly exposed to a variety of nano-products ranging from cosmetics to sports, from medical to industrial, and also space applications. With

the advent of any new revolutionary technology with enormous potential for applications, it is perhaps even more pertinent to assess the risks and challenges accompanying them. The effect of nanoparticles on biological and ecological systems in large is a subject to be studied with the highest priority. A new field, nano-toxicology, has evolved in order to probe this field. It is important to qualify the application of nanomaterials for industrial and large-scale societal applications, not only based on their properties but also based on their possible long-term side effects

\section{Conclusion}

Nanofluids are advanced fluids containing nano sized particles that have emerged during the last two decades, Nanofluids are used to enhanced system performance in many thermal engineering systems. This paper presented a review of the application of Nano fluids in solar thermal engineering\% .

\section{References}

Choi SUS Developments and applications of non-newtonian flows, in: signer DD, Wang HP, Eds. American Society of Mechanical Engineering, New York 1995:231 (66):99

Cheng L. Bandarra FEP, Thome J R. J Nanocsi Nanotech 2008.83315.

H Masuda. A Ebata. K Teramac, N. Hishinuma, Alteration of thermal conductivity and viscosity of liquid by dispersing ultra-fine particles (dispersion of 7-A1203, Si02, and Ti02 ultra-fine particles), Netsu Busesi (Japan) 7 (4) (1993) 227-233.

S.A. Putnam, D.G. Cahill, P.V. Braun, Z. Ge, R.G. Shimmin, Thermal conductivity of nanoparticle suspensions, Journal of Applied Physics 99 (8) (2006) 084308.s

M.J. Assael. C.F. Chen, I.N. Metexa, W.A. Wakeham, Thermal conductivity of suspensions of carbon nanoubes in water, international J ournal of Thermophysics 25 (4) (2004) 971-985.

C.Y.Tsai, H.T. Chien, P.P. Ding. B Chan, T.Y Luth, P.H Chen Effect of structural character of gold nanoparticle in nanofluid on heat pipe thermal performance, Material Letters 58 (20004) 14611465.

P. Keblinski J.A Esatman. D.G. Cahill, Nanofluids for thermal transport, Materials Today 8 (6) 92005) 3644.

S.M.S. Murshed, K.C Leong, C. Yang Enhanced thermal conductivity of $\mathrm{TiO}_{2}$-water based nanofluids. International J ournals of thermal sciences 44 (4) (2005) 367-373. 